\title{
基于 $\left[\mathrm{Dy}_{4}\left(\mu_{4}-0\right)\right]$ 四面体结构单元的八核和十核镝簇合物的合成、结构 及其单分子磁体行为研究
}

\author{
郭鹏虎廖小芬冷际东童明良* \\ (生物无机与合成化学教育部重点实验室 光电材料与技术国家重点实验室 中山大学化学与化学工程学院 \\ 广州 510275)
}

\begin{abstract}
摘要 通过溶剂热法合成了两个稀土簇合物, 其组成分别为: [Dy $\left.8(b p t)_{8}\left(\mu_{4}-\mathrm{O}\right)_{2}(\mu-\mathrm{OMe})_{8}\left(\mu_{1,1,3,3}-\mathrm{N}_{3}\right)\left(\mu_{1,3}-\mathrm{N}_{3}\right)\left(\mathrm{N}_{3}\right)_{2}\right] \cdot$ $11 \mathrm{H}_{2} \mathrm{O} \cdot 9 \mathrm{MeOH}(\mathbf{1})$ 和 $\left[\mathrm{Dy}_{10}(\mathrm{bpt})_{6}\left(\mu_{4}-\mathrm{O}\right)_{4}\left(\mu_{3}-\mathrm{OMe}\right)_{4}(\mu-\mathrm{OMe})_{8}(\mu-\mathrm{OAc})_{2}(\mathrm{OAc})_{2}\right] \cdot 40 \mathrm{H}_{2} \mathrm{O}(2)(\mathrm{Hbpt}=3,5$-双 (2-吡啶基)-1,2,4-三 氮唑). 1 的簇核结构由两个 $\left[\mathrm{Dy}_{4}\left(\mu_{4}-\mathrm{O}\right)\right.$ ] 四面体通过罕见的 $\mu_{1,1,3,3}$ 和 $\mu_{1,3}$ 叠氮桥连接而成, 2 则由四个共边连接的 $\left[\mathrm{Dy}_{4}\left(\mu_{4}-\mathrm{O}\right)\right]$ 四面体组成. 两个簇合物都表现出单分子磁体行为, 其中 2 是核数最高的纯稀土单分子磁体.

关键词 单分子磁体; 镝; 弛豫; 簇合物; 叠氮
\end{abstract}

\section{Synthesis, Structures and Single-Molecule Magnet Behaviour of Octanuclear and Decanuclear Dysprosium Clusters Based on $\left[\mathrm{Dy}_{4}\left(\mu_{4}-0\right)\right]$ Tetrahedral Subunits}

\author{
Guo, Penghu Liao, Xiaofen Leng, Jidong Tong, Mingliang* \\ (Key Laboratory of Bioinorganic and Synthetic Chemistry of Ministry of Education/State Key Laboratory of Optoelectronic \\ Materials and Technologies, School of Chemistry and Chemical Engineering, Sun Yat-Sen University, Guangzhou 510275,
} China)

\begin{abstract}
Two novel $\left[\mathrm{Dy}_{4}\left(\mu_{4}-\mathrm{O}\right)\right]$ based dysprosium(III) clusters, namely, [Dy $8(\mathrm{bpt})_{8}\left(\mu_{4}-\mathrm{O}\right)_{2}(\mu-\mathrm{OMe})_{8}\left(\mu_{1,1,3,3}-\mathrm{N}_{3}\right)\left(\mu_{1,3}-\mathrm{N}_{3}\right)-$ $\left.\left(\mathrm{N}_{3}\right)_{2}\right] \cdot 11 \mathrm{H}_{2} \mathrm{O} \cdot 9 \mathrm{MeOH}(\mathbf{1})$ and $\left[\mathrm{Dy}_{10}(\mathrm{bpt})_{6}\left(\mu_{4}-\mathrm{O}\right)_{4}\left(\mu_{3}-\mathrm{OMe}\right)_{4}(\mu-\mathrm{OMe})_{8}(\mu-\mathrm{OAc})_{2}(\mathrm{OAc})_{2}\right] \cdot 40 \mathrm{H}_{2} \mathrm{O}(2)(\mathrm{Hbpt}=3,5$-bis $($ pyridin2-yl)-1,2,4-trizole), are synthesized successfully. To obtain complex 1, a mixture of $\mathrm{DyCl}_{3}(0.1 \mathrm{mmol}), 2,2$ '-Hbpt $(0.1 \mathrm{mmol})$ and $\mathrm{NaN}_{3}(0.15 \mathrm{mmol})$ was sealed in a $25 \mathrm{~mL}$ vessel and then heated at $160{ }^{\circ} \mathrm{C}$ in methanol condition $(8 \mathrm{~mL})$ for $72 \mathrm{~h}$. The reaction of $\mathrm{Dy}(\mathrm{OAc})_{3}(0.17 \mathrm{mmol})$ and 2,2'-Hbpt $(0.1 \mathrm{mmol})$ under the same condition as complex 1 yielded complex 2. Single-crystal X-ray diffraction reveals that complex 1 consists of a pair of $\left[\mathrm{Dy}_{4}\left(\mu_{4}-\mathrm{O}\right)\right]$ tetrahedral units bridged by two azido groups in $\mu_{1,1,3,3}$ and $\mu_{1,3}$ modes as well as two bpt ligands, while complex 2 possesses four edge-sharing [Dy $y_{4}\left(\mu_{4}-\mathrm{O}\right)$ ] tetrahedral units. Magnetic susceptibility of polycrystalline samples were carried out using a SQUID magnetometer in the temperature range $2 \sim 300 \mathrm{~K}$ at 500 Oe dc field. The $\chi_{M} T$ value are $107.8 \mathrm{~cm}^{3} \cdot \mathrm{K}^{\prime} \mathrm{mol}^{-1}$ for complex 1 and $133.6 \mathrm{~cm}^{3} \cdot \mathrm{K} \cdot \mathrm{mol}^{-1}$ for complex 2 at $300 \mathrm{~K}$, which are close to the expected values. The maximum values of magnetization at $1.8 \mathrm{~K}$ are $40.81 \mathrm{~N} \beta$ and $51.62 \mathrm{~N} \beta$ for $\mathbf{1}$ and 2, slightly smaller than the expected saturation values. For further investigation of the dynamic behaviour, ac susceptibility measurements were undertaken under zero-dc field between $1 \sim 1500 \mathrm{~Hz}$, they both show temperature dependence and have maximum values in the $\chi_{\mathrm{M}}{ }^{\prime \prime}$ vs $v$ plots above $1.8 \mathrm{~K}$, indicating the presence of slow relaxation of the magnetization. After linear fitting the first four points of $\ln (\tau / \mathrm{s}) \mathrm{vs} 1 / T$ plot, we get energy barrier $U_{\text {eff }}=9.83(9) \mathrm{K}$, pre-exponential factor $\tau_{0}=1.63(2) \times 10^{-5} \mathrm{~s}$ for $\mathbf{1}$, and $U_{\text {eff }}=12.05(2) \mathrm{K}, \tau_{0}=6.75(8) \times 10^{-7} \mathrm{~s}$ for 2 respectively. The bridging mode of azido in complex $\mathbf{1}$ is novel and complex $\mathbf{2}$ holds the record for highest nuclearity among the reported pure lanthanide single-molecule magnets (SMMs) under zero dc field.
\end{abstract}

Keywords single-molecule magnet; dysprosium; relaxation; cluster; azido

\section{1 引言}

单分子磁体在高密度信息存储、量子计算等方面有 极为重要的应用前景 ${ }^{[1]}$. 自 1993 年第一例单分子磁体十 二核锰族合物被报道以来 ${ }^{[2]}$, 多核金属族合物就一直备 受关注, 成为当前合成化学、材料和物理等学科的热点
研究对象之一. 各国科研人员围绕单分子磁体的基态自 旋 $\left(S_{\mathrm{T}}\right)$ 、磁各向异性 $(D)$ 以及阻塞温度 $\left(T_{\mathrm{B}}\right)$ 这三个主要核 心要素开展了大量的理论和实验研究, 取得了重要的进 展 ${ }^{[3 \sim 5]}$. 单分子磁体已从第一代的 $3 \mathrm{~d}$ 金属簇合物, 第二 代的 $3 \mathrm{~d} \sim 4 \mathrm{f}$ 金属簇合物, 发展到第三代的 $4 \mathrm{f}$ 金属簇(配) 合物，单分子磁体的自旋翻转能垒相应地从第一代

*E-mail: tongml@mail.sysu.edu.cn

Received November 5, 2012; published December 6, 2012.

Project supported by the "973 Project" (2012CB821704) and the National Natural Science Foundation of China (Nos. 91122032,90922009 and 21121061). 项目受 973 计划(No. 2012CB821704)和国家自然科学基金(Nos. 91122032, 90922009, 21121061)资助. 
$\left\{\mathrm{Mn}_{12}\right\}$ 的 $66 \mathrm{~K}$ 提高到 $\left\{\mathrm{Tb}(\mathrm{Pc})_{2}\right\}$ 的 $800 \mathrm{~K}^{[6]}$, 阻塞温度也 从第一代的 $7 \mathrm{~K}$ 左右提高到第三代的接近液氮温度 ${ }^{[7]}$. 但是，目前 $4 \mathrm{f}$ 金属簇(配)合物单分子磁体的能垒都是通 过实验测得的, 无统一的理论指导该类分子磁体能垒的 计算. 同时, $4 \mathrm{f}$ 金属簇(配)合物的单分子磁体行为与分 子的局部及整体的对称性密切相关 ${ }^{[8]}$. 到目前为止, 尽 管很多稀土簇合物表现出磁化强度慢的弛豫行为, 但是 大多数的交流磁化率虚部 $\left(\chi^{\prime \prime}\right)$ 在 $2 \mathrm{~K}$ 以上都没有峰值出 现 ${ }^{[9]}$. 文献中报道的阻塞温度 $T_{\mathrm{B}}$ 在 $2 \mathrm{~K}$ 以上的 $4 \mathrm{f}$ 金属配 合物单分子磁体主要是二至八核族合物，包括: $\left\{\mathrm{Dy}_{2}\right\}^{[10]}$; 三角形和线性结构的 $\left\{\mathrm{Dy}_{3}\right\}^{[4,11]}$; 线性 ${ }^{[12]}$ 、四边 形 ${ }^{[13]}$ 、四面体 ${ }^{[14]}$ 、缺位立方烷型结构的 $\left\{\mathrm{Dy}_{4}\right\}^{[15]}$; 金字 塔形的 $\left\{\mathrm{Dy}_{5}\right\}^{[5]}$; 三角双雉形的 $\left\{\mathrm{Dy}_{6}\right\}^{[16]}$; 立方烷型结构 的 $\left\{\mathrm{Dy}_{8}\right\}^{[17]}$. 其中 $\left\{\mathrm{Dy}_{8}\right\}$ 是迄今报道的 $4 \mathrm{f}$ 单分子磁体中 核数最高的.

3,5-双(2-吡啶基)-1,2,4-三氮唑(2,2'-Hbpt) 是潜在的 含氮桥连配体, 我们曾系统地开展了基于 $2,2^{\prime}-\mathrm{Hbpt}$ 配体 的过渡金属族(配)合物研究, 并推广到稀土族合物的合 成与磁性研究中 ${ }^{[18]}$, 发现了一例在基态具有环四边形 磁矩的单分子磁体 $\left\{\mathrm{Dy}_{4}\right\}^{[19]}$. 为了深化 Dy(III)-bpt 体系 的研究, 我们通过引入羧酸根、叠氮根等多种桥连配体, 合成出两个结构新颖的八核和十核镝簇合物: $\left[\mathrm{Dy}_{8}(\mathrm{bpt})_{8}\left(\mu_{4}-\mathrm{O}\right)_{2}(\mu-\mathrm{OMe})_{8}\left(\mu_{1,1,3,3}-\mathrm{N}_{3}\right)\left(\mu_{1,3}-\mathrm{N}_{3}\right)\left(\mathrm{N}_{3}\right)_{2}\right] \bullet 11 \mathrm{H}_{2} \mathrm{O} \bullet$ $9 \mathrm{MeOH} \quad(1)$ 和 $\left[\mathrm{Dy}_{10}(\mathrm{bpt})_{6}\left(\mu_{4}-\mathrm{O}\right)_{4}\left(\mu_{3}-\mathrm{OMe}\right)_{4}(\mu-\mathrm{OMe})_{8}-\right.$ $\left.(\mu-\mathrm{OAc})_{2}(\mathrm{OAc})_{2}\right] \cdot 40 \mathrm{H}_{2} \mathrm{O}(2)(\mathrm{Hbpt}=3,5$-bis(pyridin-2-yl)1,2,4-trizole). 这里我们报道两个族合物的合成、结构及 其单分子磁体行为.

\section{2 结果与讨论}

通过 X-射线单晶衍射测试, 配合物 1 和 2 分子都不

(a)
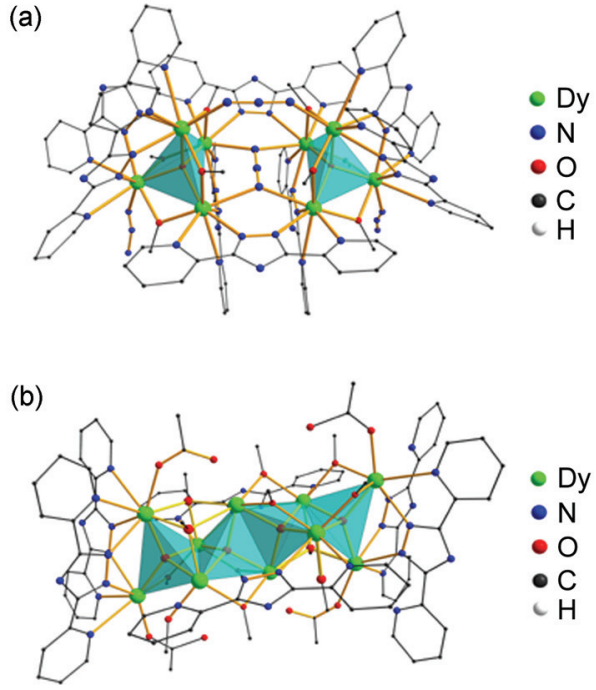

带电荷, 其晶体学数据如表 1 所示. 配合物 1 结晶于单 斜晶系, $C 2 / c$ 空间群，分子是由两个等同的 $\left[\mathrm{Dy}_{4}\left(\mu_{4}-\mathrm{O}\right)\right]$ 四面体通过 $\mu_{1,1,3,3}$ 和 $\mu_{1,3}$ 叠氮桥(图 1a)以及两个 bpt 配体 连接起来, 所有镝离子都位于四面体的顶角位置. 在四 面体 $\left[\mathrm{Dy}_{4}\left(\mu_{4}-\mathrm{O}\right)\right]$ 单元内, $\mathrm{Dy}-\mathrm{O}$ 键长处在 $2.32 \sim 2.37 \AA$ 范围, 四面体的四个边由 $\mu-\mathrm{OMe}^{-}$连接起来, 另外两个 边通过 bpt 配体三氮唑桥连接, Dy $\cdots$ Dy 距离在 3.67 $4.13 \AA$ 范围。两个四面体间的 $\mathrm{Dy}(3) \cdots \mathrm{Dy}(3 \mathrm{~A})$ 和 $\operatorname{Dy}(1) \cdots \operatorname{Dy}(2 \mathrm{~A})$ 距离分别是 $6.25 \AA$ 和 $4.78 \AA$. 与 Ishikawa 课题组 ${ }^{[7]}$ 之前报道的单离子磁体 $\left[\mathrm{Pc}_{2} \mathrm{Ln}\right]$ 一样, 配合物 $\mathbf{1}$ 的所有镝离子都采用变形的四方反棱柱配位几 何(图 2a). 配位原子中, 五个 $\mathrm{N}$ 原子分别来自两个 bpt 配体和一个端基配位的叠氮根, 三个氧原子分别来自于 两个 $\mu-\mathrm{OMe}^{-}$和 $\mu_{4}-\mathrm{O}^{2-}$. $\angle \mathrm{Dy} \cdots \mathrm{O} \cdots$ Dy 键角在 $101.37^{\circ} \sim$ $125.34^{\circ}$ 范围, 分子间 Dy $\cdots$ Dy 的最近距离为 $9.04 \AA$.

到目前为止，虽然报道过 $\left\{\mathrm{Dy}_{8}\right\}$ 单分子磁体 ${ }^{[17]}$ 和叠 氮桥联的过渡金属配合物 ${ }^{[20]}$ ，但是在稀土单分子磁体 里面, 像这样两个 $\left[\mathrm{Dy}_{4}\left(\mu_{4}-\mathrm{O}\right)\right]$ 四面体通过 $\mu_{1,1,3,3}$ 和 $\mu_{1,3}$ 叠 氮桥联接起来(图 3b)的结构, 之前从来没有报道过.

配合物 2 结晶于三斜晶系, $P-1$ 空间群, 由四个共边 连接的 $\left[D y_{4}\left(\mu_{4}-\mathrm{O}\right)\right]$ 四面体组成. 所有的镝离子共存在三 种配位几何(图 2): 对于 $\mathrm{Dy}(4)$ 和 $\mathrm{Dy}(5) 、 \mathrm{Dy}(1)$ 和 $\mathrm{Dy}(3)$ 、 $\mathrm{Dy}(2)$ 分别是变形的双加帽三棱柱 $\left(\mathrm{N}_{4} \mathrm{O}_{4}\right.$ 的配位环境)、 变形的单加帽三棱柱 $\left(\mathrm{N}_{2} \mathrm{O}_{5}\right.$ 配位环境)、变形的单加帽三 方反棱柱 ( $\mathrm{O}_{7}$ 的配位环境). 配合物 2 的晶体学最小独立 单元是两个共边连接的四面体 (图 $1 \mathrm{~b}$ ), 四面体 $\left\{\mathrm{Dy}_{4}\left[\mu_{4}-\mathrm{O}(1)\right]\right\}$ 的 $\mathrm{Dy}-\mathrm{O}(1)$ 键长介于 $2.27 \sim 2.30 \AA$ 之间, Dy $\cdots$ Dy 距离在 $3.50 \sim 4.14 \AA$ 之间, 六个边分别由两个 $\mu-\mathrm{OMe}^{-}$、两个 $\mu_{3}-\mathrm{OMe}^{-}$、一个 $\mu_{4}-\mathrm{O}^{2-}$ 和两个来自于 $\mathrm{bpt}$ 配体的三氮唑桥连接起来. Dy(5)还与一个端基乙酸根
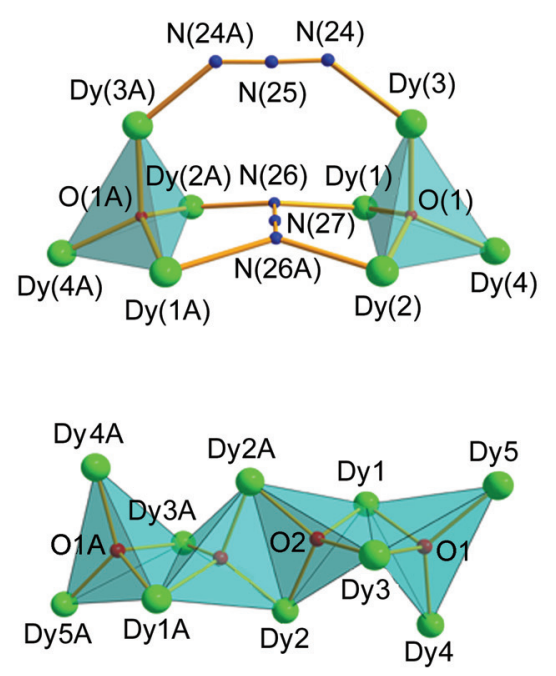

图 1 配合物 1 (a)和 2 (b)的分子结构和簇核结构

Figure 1 Molecular (left) and core (right) structures of 1 (a) and 2 (b) 
表 1 配合物 1 和 $\mathbf{2}$ 的晶体学数据

Table 1 Crystallographic data for complex 1 and complex 2

\begin{tabular}{|c|c|c|}
\hline \multirow{2}{*}{ Formula } & Complex 1 & Complex 2 \\
\hline & $\mathrm{C}_{113} \mathrm{H}_{146} \mathrm{Dy}_{8} \mathrm{~N}_{52} \mathrm{O}_{30}$ & $\mathrm{C}_{92} \mathrm{H}_{176} \mathrm{Dy}_{10} \mathrm{~N}_{30} \mathrm{O}_{64}$ \\
\hline$F_{\mathrm{w}}$ & 4012.70 & 4351.55 \\
\hline Temperature/K & 298 & 150 \\
\hline$a / \AA ̊$ & $21.0126(4)$ & $15.264(4)$ \\
\hline$b / \AA$ & $19.2248(3)$ & $15.484(4)$ \\
\hline$c / \AA ̊$ & $35.9910(8)$ & $15.757(4)$ \\
\hline$\alpha /\left(^{\circ}\right)$ & 90 & $96.943(4)$ \\
\hline$\beta /\left(^{\circ}\right)$ & $99.856(1)$ & $109.820(4)$ \\
\hline$\gamma /\left(^{\circ}\right)$ & 90 & $99.780(4)$ \\
\hline Cell volume $/ \AA^{3}$ & $14324.5(5)$ & $3388.0(14)$ \\
\hline Space group & $C 2 / c$ & $P-1$ \\
\hline$R_{1}^{a}[F>4 \sigma(F)]$ & 0.0456 & 0.0475 \\
\hline$w R_{2}{ }^{b}\left(F^{2}\right.$, all data $)$ & 0.1375 & 0.1253 \\
\hline$R_{\text {int }}$ & 0.0334 & 0.0427 \\
\hline$R_{\sigma}$ & 0.0418 & 0.0775 \\
\hline Goof & 1.056 & 0.935 \\
\hline$Z$ & 4 & 1 \\
\hline
\end{tabular}

(a)

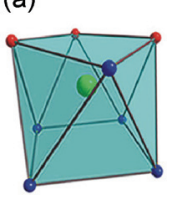

(b)

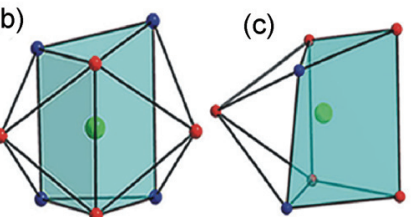

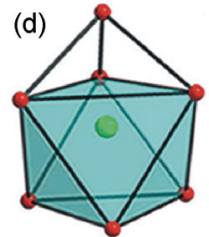

图 2 配合物 1 中镝离子的配位几何: (a) 四方反棱柱. 配合物 $\mathbf{2}$ 中镝 离子的三种配位几何: (b) 双加帽三棱柱; (c) 单加帽三棱柱; (d) 单加 帽三方反棱柱. 镝, 绿色; 氮, 蓝色; 氧, 红色

Figure 2 The coordination geometries of metal ions in complex 1: (a) square antiprism. The three coordination geometries of metal ions in complex 2: (b) bicapped trigonal prism; (c) monocapped trigonal prism; (d) monocapped trigonal antiprism. Color scheme: green, Dy; blue, N; red, $\mathrm{O}$

配位. $\angle \mathrm{Dy} \cdots \mathrm{O}(1) \cdots \mathrm{Dy}$ 键角在 $100.45^{\circ} \sim 127.96^{\circ}$ 范围. 四面体 $\left\{\mathrm{Dy}_{4}\left[\mu_{4}-\mathrm{O}(2)\right]\right\}$ 的 $\mathrm{Dy}-\mathrm{O}(2)$ 键长在 $2.20 \sim 2.25 \AA$ 之间, Dy $\cdots$ Dy 距离介于 $3.50 \sim 3.71 \AA$ 之间, 六个边分别 由两个 $\mu-\mathrm{OMe}^{-}$、两个 $\mu_{3}-\mathrm{OMe}^{-} 、 \mu_{4}-\mathrm{O}^{2-} 、 \mu_{4}-\mathrm{O}^{2-}$ 连接起 来, $\angle \mathrm{Dy} \cdots \mathrm{O}(2) \cdots \mathrm{Dy}$ 键角在 $105.08^{\circ} \sim 111.60^{\circ}$ 范围. 两 个四面体 $\left\{\mathrm{Dy}_{4}\left[\mu_{4}-\mathrm{O}(1)\right]\right\}$ 和 $\left\{\mathrm{Dy}_{4}\left[\mu_{4}-\mathrm{O}(2)\right]\right\}$ 通过共用 $\operatorname{Dy}(1) \cdots \operatorname{Dy}(3)$ 边连接起来. 相邻分子间 Dy $\cdots$ Dy 最近距 离为 $9.32 \AA$.

相比较而言, 配合物 2 不仅与文献中报道的具有慢 弛豫行为 $\left\{\mathrm{Dy}_{10}\right\}$ 族合物 ${ }^{[9 \mathrm{a}]}$ 结构不同, 更重要的是, 前者 的交流磁化率虚部在温度 $T_{\mathrm{B}}>2 \mathrm{~K}$ 时就有峰值出现, 也 是迄今为止核数最高的 $4 \mathrm{f}$ 单分子磁体.

在 $500 \mathrm{Oe}$ 外场下, $2.0 \sim 300 \mathrm{~K}$ 温度范围内, 测试了 配合物 1 和 2 的变温磁化率, 如图 $4 \chi_{\mathrm{M}} T-T$ 曲线所示. 室 (a)

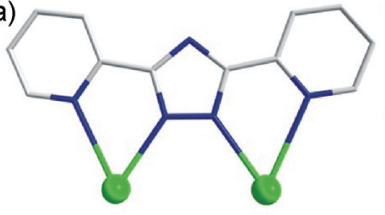

(b)

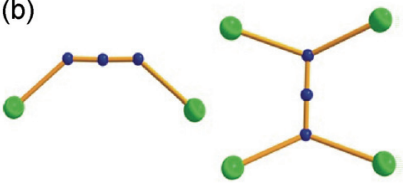

图 3 bpt 配体以及 $\mu_{1,3}$ 和 $\mu_{1,1,3,3}$ 叠氮桥的配位模式

Figure 3 Bridging modes of bpt ligand and $\mu_{1,3}$-azido and $\mu_{1,1,3,3}$-azido

温 $300 \mathrm{~K}$ 时, 配合物 $\mathbf{1}$ 和 $\mathbf{2}$ 的 $\chi_{\mathrm{M}} T$ 值分别是 107.8 $\mathrm{cm}^{3} \cdot \mathrm{K} \cdot \mathrm{mol}^{-1}$ 和 $133.6 \mathrm{~cm}^{3} \cdot \mathrm{K} \cdot \mathrm{mol}^{-1}$, 分别接近于未耦合 的八个和十个镝离子 $(S=5 / 2, L=5, J=15 / 2, g=4 / 3)$ 的 $\chi_{\mathrm{M}} T$ 值 $113.3 \mathrm{~cm}^{3} \cdot \mathrm{K} \cdot \mathrm{mol}^{-1}$ 和 $141.7 \mathrm{~cm}^{3} \cdot \mathrm{K} \cdot \mathrm{mol}^{-1[21]}$. 对于 配合物 1 来说, 随着温度的降低, $\chi_{\mathrm{M}} T$ 值缓慢减小, $30 \mathrm{~K}$ 时开始迅速减小, $2.0 \mathrm{~K}$ 时达到最小值 $32.8 \mathrm{~cm}^{3} \cdot \mathrm{K}^{\prime} \cdot \mathrm{mol}^{-1}$. 对于配合物 2 , 随着温度降低 $\chi_{\mathrm{M}} T$ 值变化不大, 直到温 度降到 $45 \mathrm{~K}$ 左右时, $\chi_{\mathrm{M}} T$ 值开始急剧减小, $2.0 \mathrm{~K}$ 时有最 小值 $61.2 \mathrm{~cm}^{3} \cdot \mathrm{K} \cdot \mathrm{mol}^{-1}$. 上述行为说明了可能是由于晶 体场作用导致的激发态斯塔克次能级(excited Stark sublevel)布居减少(depopulation)和/或者是由于反铁磁相互 作用 ${ }^{[21,22]}$. 在不同温度下测试了配合物 1 和 $\mathbf{2}$ 的变场磁 化强度曲线(图 4 内插图), $1.8 \mathrm{~K}$ 时, 配合物 $\mathbf{1}$ 和 $\mathbf{2}$ 的磁 化强度最大值分别是 $40.81 \mathrm{~N} \beta$ 和 $51.62 \mathrm{~N} \beta$, 与理论值 $41.84 \mathrm{~N} \beta$ 和 $52.30 \mathrm{~N} \beta$ 接近. 不同温度下的 $M \sim H \bullet T^{-1}$ 曲 线既没有达到饱和，也没有重合，表明存在显著的磁各 向异性或者低能量的激发态布居 ${ }^{[17]}$.

为了进一步研究其磁行为, 在零场的条件下, 1 $1500 \mathrm{~Hz}$ 范围内，测试了不同温度下配合物 $\mathbf{1}$ 和 $\mathbf{2}$ 的交 流磁化率，分别如图 5 左右两边所示. 在 $1.8 \mathrm{~K}$ 以上, 交 流磁化率信号都具有很强的频率依赖, 并且在虚部 $\left(\chi^{\prime \prime}\right)$ 出现峰值, 表明此时弛豫作用的频率 $(1 / \tau)$ ( $\tau$ 是弛豫时间) 等于交变场的频率 $(2 \pi v)$, 这些行为都是单分子磁体的 典型特征.

以 $\ln (\tau / \mathrm{s})$ 对 $T^{-1}$ 做图, $[\tau=1 /(2 \pi v)], v$ 是不同温度 $T$ 下 $\chi_{\mathrm{M}}{ }^{\prime}$ 峰值对应的交流磁场频率), 如图 6 所示. 按照阿 伦尼乌斯公式 $\tau=\tau_{0} \exp \left(U_{\text {eff }} / k_{\mathrm{B}} T\right)$, 对 $\ln (\tau / \mathrm{s})-T^{-1}$ 曲线进行 线性拟合，得到配合物 1 和 2 的有效能垒 $\left(U_{\text {eff }}\right)$ 和指前因 子 $\left(\tau_{0}\right)$ 分别是 $U_{\text {eff }}=9.83(9) \mathrm{K}, \tau_{0}=1.63 \times 10^{-5} \mathrm{~s}$ 和 $U_{\mathrm{eff}}=$ $12.05(2) \mathrm{K}, \tau_{0}=6.75(8) \times 10^{-7} \mathrm{~s}$.

以不同温度下的 $\chi_{\mathrm{M}}{ }^{\prime}$ 对 $\chi_{\mathrm{M}}{ }^{\prime}$ 作图得到近似于半圆形 的 Cole-Cole 图(图 7), 按照德拜模型(Debye model)对这 些数据进行非线性拟合，得到配合物 1 和 $\mathbf{2}$ 的弛豫时间

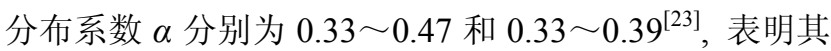
弛豫时间分布相对较宽. 为了排除自旋玻璃的可能性, 根据公式 $\phi=\left(\Delta T_{\mathrm{p}} / T_{\mathrm{p}}\right) / \Delta(\log v)\left(T_{\mathrm{p}}\right.$ 是指相应 $\chi^{\prime \prime}$ 的峰值温度, $v$ 是指不同温度下 $\chi^{\prime \prime}$ 峰值对应的交流磁场频率), 计算得 到配合物 1 和 2 的参数 $\phi$ 分别为 $0.53 \sim 0.59$ 和 $0.35 \sim 0.56$, 超出文献中报道的自旋玻璃 $0.01<\phi<0.08$ 的范围 ${ }^{[24]}$. 

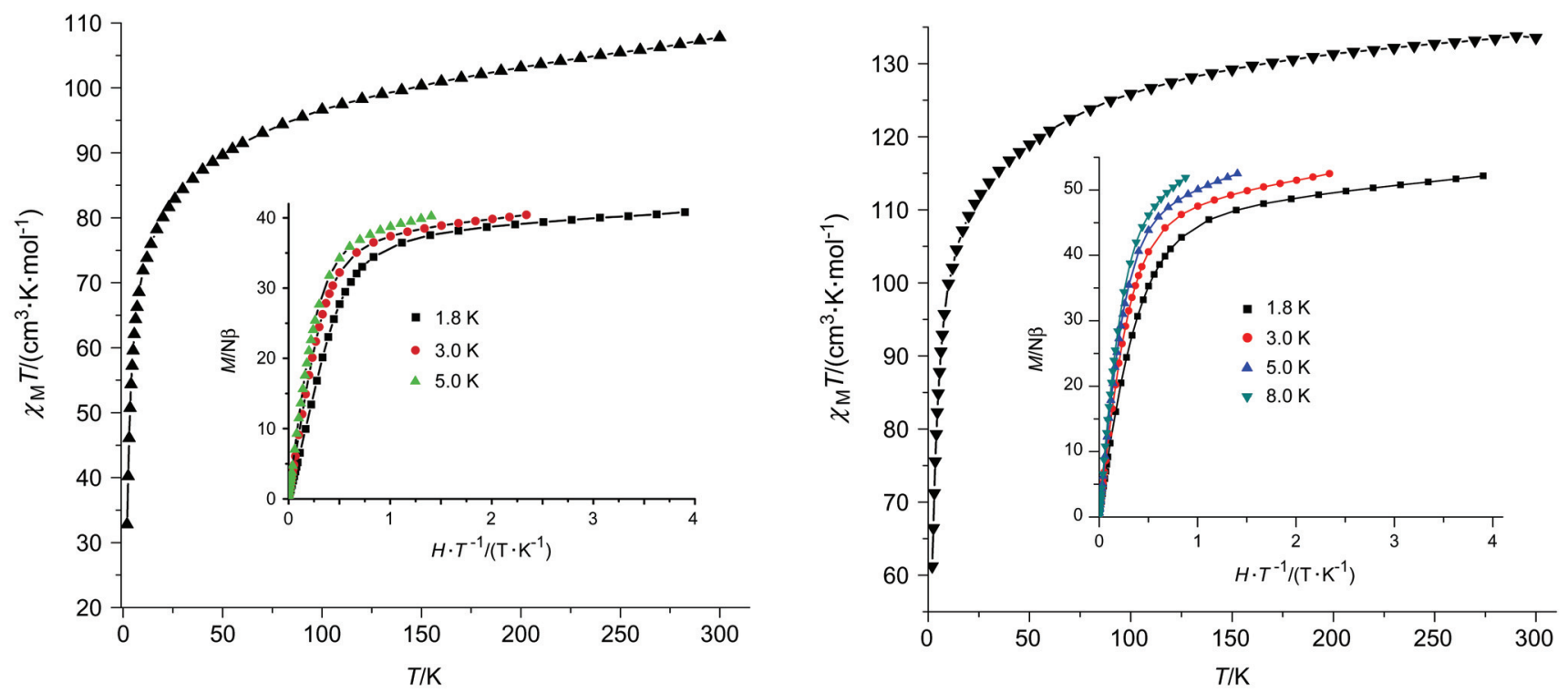

图 4 配合物 $\mathbf{1}$ (左边)和 $\mathbf{2}$ (右边)在 $500 \mathrm{Oe}$ 外场下的 $\chi_{\mathrm{M}} T \mathrm{vs} T$ 图和 $M \mathrm{vs} H \cdot T^{-1}$ 图(内插部分)

Figure 4 The $\chi_{\mathrm{M}} T$ vs $T$ plots at 500 Oe and $M$ vs $H \bullet T^{-1}$ plots (inset) for complex 1 (left) and 2 (right)
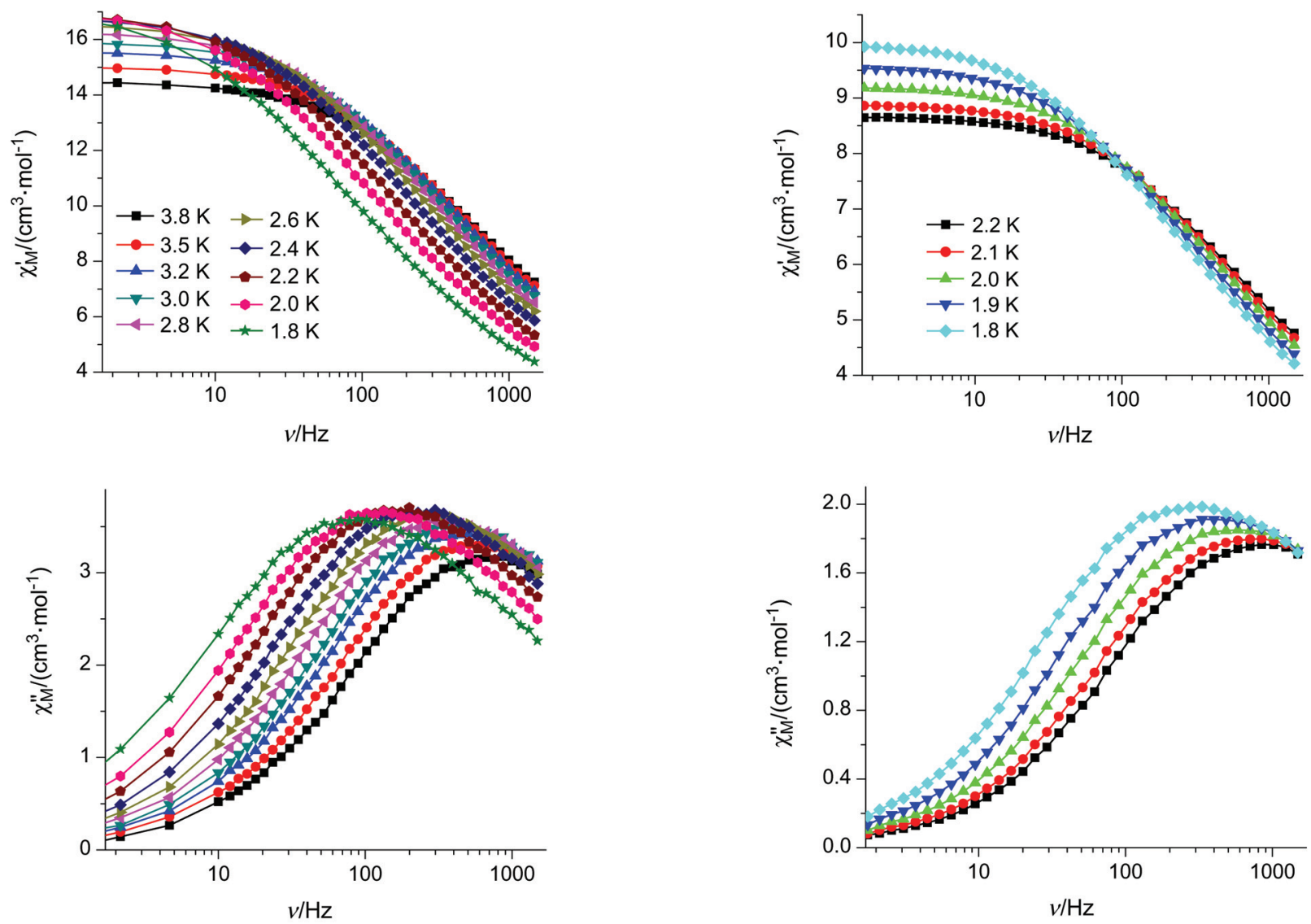

图 5 零外场条件下, 配合物 $\mathbf{1}$ (左边) 和 $\mathbf{2}$ (右边)在不同温度下实部和虚部交流磁化率信号随频率的变化

Figure 5 Frequency dependence of the in-phase $\chi^{\prime}$ (top) and out-of-phase $\chi^{\prime \prime}$ (bottom) components of ac susceptibility for complex 1 (left) and 2 (right) under zero-dc field

\section{3 结论}

在本研究中，通过 3,5-双(2-吡啶基)-1,2,4-三氮唑 (Hbpt)配体和稀土盐的溶剂热反应，合成了两个新颖的
镝簇合物，其组成分别为: $\left[\mathrm{Dy}_{8}(\mathrm{bpt})_{8}\left(\mu_{4}-\mathrm{O}\right)_{2}(\mu-\mathrm{OMe})_{8}-\right.$ $\left.\left(\mu_{1,1,3,3}-\mathrm{N}_{3}\right)\left(\mu_{1,3}-\mathrm{N}_{3}\right)\left(\mathrm{N}_{3}\right)_{2}\right] \cdot 11 \mathrm{H}_{2} \mathrm{O} \cdot 9 \mathrm{MeOH}(\mathbf{1})$ 和 $\left[\mathrm{Dy}_{10}(\mathrm{bpt})_{6}-\right.$ $\left.\left(\mu_{4}-\mathrm{O}\right)_{4}\left(\mu_{3}-\mathrm{OMe}\right)_{4}(\mu-\mathrm{OMe})_{8}-(\mu-\mathrm{OAc})_{2}(\mathrm{OAc})_{2}\right] \cdot 40 \mathrm{H}_{2} \mathrm{O} \quad(2)$. 

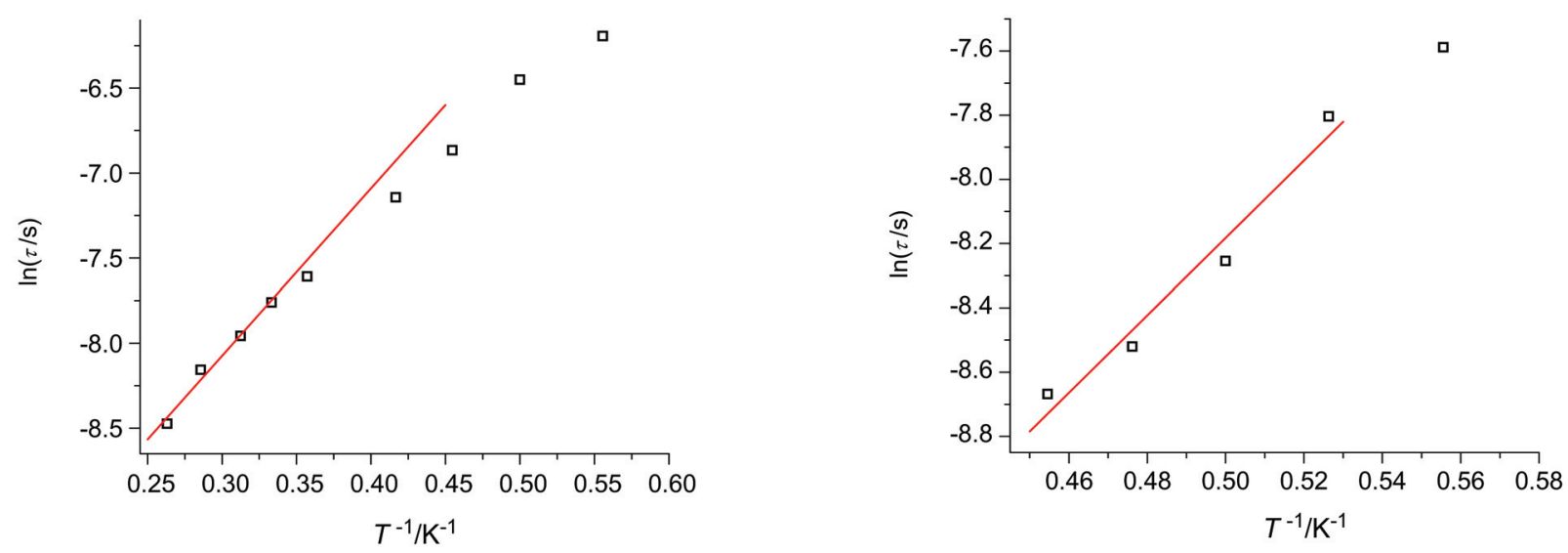

图 6 在零外场下, 配合物 $\mathbf{1}$ (左边) 和 $\mathbf{2}$ (右边)对数弛豫时间 $\ln (\tau / \mathrm{s})$ 对 $T^{-1}$ 做图, 红色实线为 Arrhenius 公式拟合

Figure 6 Magnetization relaxation time, $\ln (\tau / \mathrm{s})$ vs $T^{-1}$ plot under $H_{\mathrm{dc}}=0$, The red line is fitted with the Arrhenius law
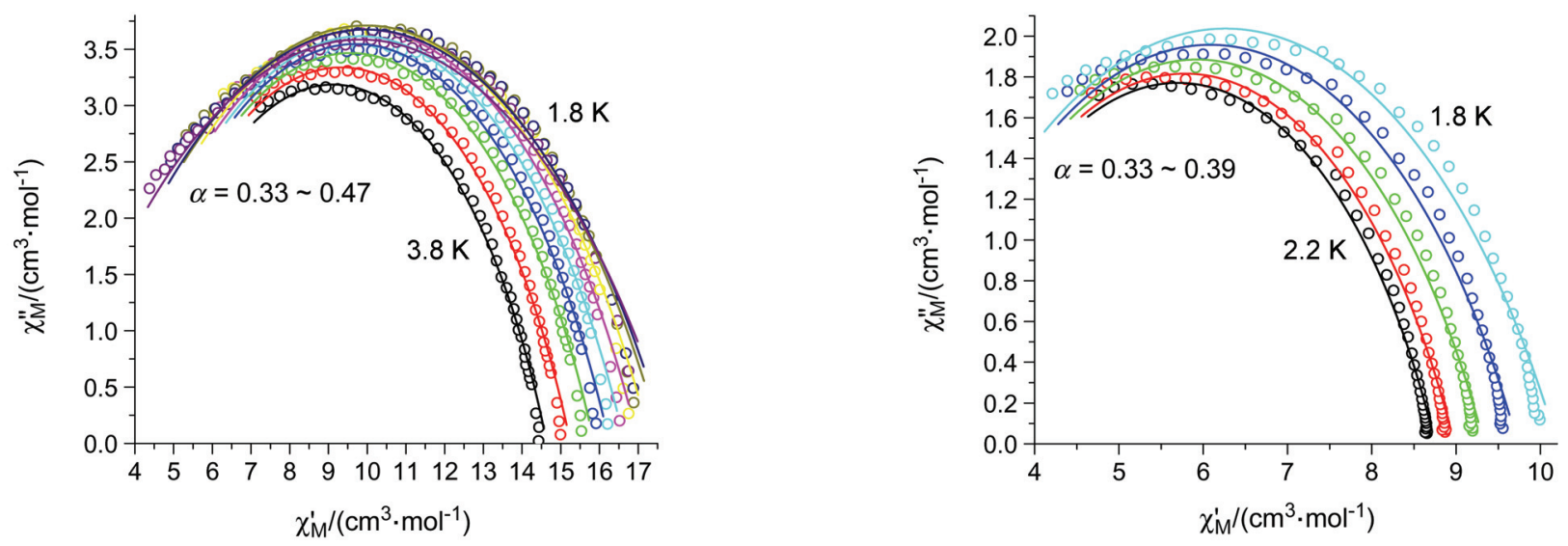

图 7 以交流磁化率的 $\chi$ 对 $\chi^{\prime \prime}$ 作图得到了配合物 $\mathbf{1}$ (左边)和 $\mathbf{2}$ (右边)的 Cole-Cole 图. 实线代表用德拜模型进行的最佳拟合

Figure 7 Cole-Cole plots for complex 1 (left) and 2 (right), obtained from variable-frequency ac susceptibility data under a zero dc field in the temperature range of $1.8 \sim 3.8 \mathrm{~K}$ (left) and $1.8 \sim 2.2 \mathrm{~K}$ (right) respectively. Solid lines represent best fits to the data using a generalized Debye model

1 的簇核结构由两个 $\left[\mathrm{Dy}_{4}\left(\mu_{4}-\mathrm{O}\right)\right]$ 四面体通过罕见的 $\mu_{1,1,3,3}$ 和 $\mu_{1,3}$ 叠氮桥连接而成, 2 则由四个共边连接的 $\left[\mathrm{Dy}_{4}\left(\mu_{4}-\mathrm{O}\right)\right]$ 四面体组成. 两个簇合物都表现出单分子磁 体行为, 其中 2 是核数最高的纯稀土单分子磁体.

\section{4 实验部分}

4.1 配 合 物 $\left[\mathrm{Dy} \mathrm{y}_{8}(\mathrm{bpt})_{8}\left(\mu_{4}-\mathrm{O}\right)_{2}(\mu-\mathrm{OMe})_{8}\left(\mu_{1,1,3,3}-\mathrm{N}_{3}\right)-\right.$ $\left.\left(\mu_{1,3}-\mathrm{N}_{3}\right)\left(\mathrm{N}_{3}\right)_{2}\right] \cdot 11 \mathrm{H}_{2} \mathrm{O} \cdot 9 \mathrm{MeOH}$ (1)的合成

将 $0.037 \mathrm{~g}$ 的 $\mathrm{DyCl}_{3} \cdot 6 \mathrm{H}_{2} \mathrm{O}, 0.023 \mathrm{~g} 2,2^{\prime}-\mathrm{Hbpt}$ 和 0.010 $\mathrm{g} \mathrm{NaN}_{3}$ 混合溶于 $8 \mathrm{~mL}$ 分析纯甲醇, 然后密封在 $25 \mathrm{~mL}$ 特氟龙(Teflon)做内祄的不锈钢容器里, 在 $160{ }^{\circ} \mathrm{C}$ 条件 下恒温 $72 \mathrm{~h}$, 然后降至室温, 得到无色透明的块状晶体, 按 Dy 计算产率约 10\%. IR (KBr) v: 3410br, 2082m, $2037 \mathrm{w}, 1797 \mathrm{w}, 1603 \mathrm{~s}, 1565 \mathrm{w}, 1497 \mathrm{w}, 1141 \mathrm{~s}, 1285 \mathrm{w}$, 1054m, 876w, 802w, 747w, 728w, 631w, 462 w cm ${ }^{-1}$. Anal. calcd for C 33.82, H 3.67, N 18.15; found C 33.63, H 3.53, N 17.98.

\section{2 配 合物 $\left[\mathrm{Dy}_{10}(\mathrm{bpt})_{6}\left(\mu_{4}-\mathrm{O}\right)_{4}\left(\mu_{3}-\mathrm{OMe}\right)_{4}(\mu-\mathrm{OMe})_{8^{-}}\right.$ $\left.(\mu-\mathrm{OAc})_{2}(\mathrm{OAc})_{2}\right] \cdot 40 \mathrm{H}_{2} \mathrm{O}(2)$ 的合成}

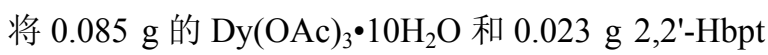
溶解于 $8 \mathrm{~mL}$ 分析纯甲醇, 然后密封在 $25 \mathrm{~mL}$ 特氟龙做 内祄的不锈钢容器里, 在 $160{ }^{\circ} \mathrm{C}$ 条件下恒温 $72 \mathrm{~h}$, 降至 室温, 得到无色透明的块状晶体, 按 Dy 计算产率约 $37 \%$. IR (KBr) v: 3388br, 1604m, 1569m, 1498w, 1466m, $1410 \mathrm{~s}, 1282 \mathrm{w}, 1186 \mathrm{w}, 1152 \mathrm{w}, 1096 \mathrm{w}, 1054 \mathrm{w}, 1016 \mathrm{w}$, $803 \mathrm{w}, 747 \mathrm{~m}, 731 \mathrm{~m}, 663 \mathrm{w}, 630 \mathrm{w}, 472 \mathrm{w} \mathrm{cm} \mathrm{cm}^{-1}$. Anal. calcd for C 25.39, H 4.08, N 9.66; found C 25.50, H 4.25, N 9.51 .

\section{References}

[1] Leuenberger, M. N.; Loss, D. Nature 2001, 410, 789.

[2] (a) Sessoli, R.; Gatteschi, D.; Caneschi, A.; Novak, M. A. Nature 1993, 365, 141; (b) Sessoli, R.; Tsai, H. L.; Schake, A. R.; Wang, S.; Vincent, J. B.; Folting, K.; Gatteschi, D.; Christou, G.; Hendrickson, D. N. J. Am. Chem. Soc. 1993, 115, 1804.

[3] Mondal, K. C.; Sundt, A.; Lan, Y.; Kostakis, G. E.; Waldmann, O.; Ungur, L.; Chibotaru, L. F.; Anson, C. E.; Powell, A. K. Angew. 
Chem. Int. Ed. 2012, 51, 7550.

[4] (a) Tang, J.; Hewitt, I.; Madhu, N. T.; Chastanet, G.; Wernsdorfer, W.; Anson, C. E.; Benelli, C.; Sessoli, R.; Powell, A. K. Angew. Chem., Int. Ed. 2006, 45, 1729; (b) Chibotaru, L. F.; Ungur, L.; Soncini, A. Angew. Chem., Int. Ed. 2008, 47, 4126.

[5] (a) Gamer, M. T.; Lan, Y.; Roesky, P. W.; Powell, A. K.; Clérac, R. Inorg. Chem. 2008, 47, 6581; (b) Blagg, R. J.; Muryn, C. A.; McInnes, E. J. L.; Tuna, F.; Winpenny, R. E. P. Angew. Chem., Int. Ed. 2011, 50, 6530.

[6] Francesca, B.; Carretta, P.; Marta, F.; Giorgio, Z.; Michael, J. G.; Jose, R. G.-M.; Olaf, F.; Susan, B.; Mario, R. J. Am. Chem. Soc. 2009, 131, 4387.

[7] Ishikawa, N.; Ishikawa, M. T.; Koshihara, S.; Kaizu, Y. J. Am. Chem. Soc. 2003, 125, 8694.

[8] Liu, J.-L.; Yuan, K.; Leng, J.-D.; Ungur, L.; Wernsdorfer, W.; Guo, F.-S.; Chibotaru, L.-F.; Tong, M.-L. Inorg. Chem. 2012, 51, 8538.

[9] (a) Ke, H.; Xu, G. F.; Zhao, L.; Tang, J.; Zhang, X. Y.; Zhang, H. J.; Chem. Eur. J. 2009, 15, 10335; (b) Miao, Y. L.; Liu, J. L.; Leng, J. D.; Lin, Z. J.; Tong, M. L. CrystEngComm 2011, 13, 3345.

[10] (a) Lin, P. H.; Burchell, T. J.; Clérac, R.; Murugesu, M. Angew. Chem., Int. Ed. 2008, 47, 8848; (b) Xu, G. F.; Wang, Q. L.; Gamez, P.; Ma, Y.; Cléra, R.; Tang, J.; Yan, S. P.; Cheng, P.; Liao, D. Z. Chem. Commun 2010, 46, 1506.

[11] (a) Hewitt, I. J.; Lan, Y.; Anson, C. E.; Luzon, J.; Sessoli, R.; Powell, A. K. Chem. Commun. 2009, 6765; (b) Guo, F. S.; Liu, J. L.; Leng, J. D.; Meng, Z. S.; Lin, Z. J.; Tong, M. L.; Gao, S.; Ungur, L.; Chibotaru, L. F. Chem. Eur. J. 2011, 17, 2458; (c) Muhammad, A. U.; Santokh, S. T.; Louise, N. D.; Fatemah, H.; Muralee, M.; Laurence, K. T. Inorg. Chem. 2012, 51, 1028.

[12] (a) Guo, Y. N.; Xu, G. F.; Gamez, P.; Zhao, L.; Lin, S. Y.; Deng, R.; Tang, J.; Zhang, H. J. J. Am. Chem. Soc. 2010, 132, 8538; (b) Lin, S. Y.; Zhao, L.; Ke, H.; Guo, Y. N.; Tang, J.; Guo, Y.; Dou, J. Dalton Trans. 2012, 41, 3248.

[13] (a) Zheng, Y. Z.; Lan, Y.; Anson, C. E.; Powell, A. K. Inorg. Chem. 2008, 47, 10813; (b) Lin, P. H.; Burchell, T. J.; Ungur, L.; Chibotaru, L. F.; Wernsdorfer, W.; Murugesu, M. Angew. Chem., Int. Ed. 2009, 48, 9489; (c) Bi, Y.; Wang, X. T.; Liao, W.; Wang, X.; Deng, R.; Zhang, H.; Gao, S. Inorg. Chem. 2009, 48, 11743; (d) Abbas, G.; Lan, Y.; Kostakis, G. E.; Wernsdorfer, W.; Anson, C. E.; Powell, A.
K. Inorg. Chem. 2010, 49, 8067; (e) Yan, P. F.; Lin, P. H.; Habib, F.; Aharen, T.; Murugesu, M.; Deng, Z. P.; Li, G. M.; Sun, W. B. Inorg. Chem. 2011, 50, 7059; (f) Xue, S.; Zhao, L.; Guo, Y. N.; Tang, J. Dalton Trans. 2012, 41, 351; (g) Guo, F.-S.; Guo, P.-H.; Meng, Z.-S.; Tong, M.-L. Polyhedron 2011, 30, 3079; (h) Miao, Y.-L.; Liu, J.-L.; Li, J.-Y.; Leng, J.-D.; Ou, Y.-C.; Tong, M.-L. Dalton Trans. 2011, 40, 10229; (i) Guo, F.-S.; Liu, J.-L.; Leng, J.-D.; Tong, M.-L.; Gao, S.; Ungur, L.; Chibotaru, L. F. Chem. Eur. J. 2011, 17, 2458; (j) Leng, J.-D.; Liu, J.-L.; Zheng, Y.-Z.; Ungur, L.; Chibotaru, L. F.; Guo, F.-S.; Tong, M.-L. Chem. Commun. 2013, 49, 158.

[14] Lin, P. H.; Korokov, I.; Wernsdorfer, W.; Ungur, L.; Chibotaru, L. F.; Murugesu, M. Eur. J. Inorg. Chem. 2011, 1535.

[15] Ke, H.; Gamez, P.; Zhao, L.; Xu, G. F.; Xue, S.; Tang, J. Inorg. Chem. 2010, 49, 7549.

[16] Tian, H.; Wang, M.; Zhao, L.; Guo, Y. N.; Guo, Y.; Tang, J.; Liu, Z. Chem. Eur. J. 2012, 18, 442.

[17] Tian, H.; Zhao, L.; Guo, Y. N.; Guo, Y.; Tang, J.; Liu, Z. Chem. Commun. 2012, 48, 708 .

[18] Bao, X.; Leng, J. D.; Meng, Z. S.; Lin, Z. J.; Tong, M. L.; Nihei, M.; Oshio, H. Chem. Eur. J. 2010, 16, 6196.

[19] (a) Guo, P. H.; Liu, J. L.; Zhang, Z. M.; Ungur, L.; Chibotaru, L. F.; Leng, J. D.; Guo, F. S.; Tong, M. L. Inorg. Chem. 2012, 51, 1233; (b) Zhang, Z.-M.; Guo, F.-S.; Guo, P.-H.; Liu, J.-L.; Tong, M.-L. Sci. China Chem. 2012, 55, 934

[20] Wang, X. Y.; Wang, Z. M.; Gao, S. Chem. Commun. 2008, 281.

[21] (a) Kahn, O. Molecular Magnetism, VCH Publishers, New York, 1993; (b) Sutter, J. P.; Kahn, M. L. Magnetism: Molecules to Materials, Vol. 5, Eds.: Miller, J. S.; Drillon, M., Wiley VCH, Weiheim, 2005, p. 161.

[22] (a) Ishikawa, N.; Sugita, M.; Wernsdorfer, W. J. Am. Chem. Soc. 2005, 127, 3650; (b) AlDamen, M. A.; Clemente-Juan, J. M.; Coronado, E.; Marti-Gastaldo, C.; Gaita-Arino, A. J. Am. Chem. Soc. 2008, 130, 8874; (c) Jiang, S. D.; Wang, B. W.; Sun, H. L.; Wang, Z. M.; Gao, S. J. Am. Chem. Soc. 2011, 133, 4730; (d) Bi, Y.; Guo, Y. N.; Zhao, L.; Guo, Y.; Lin, S. Y.; Jiang, S. D.; Tang, J.; Wang, B. W.; Gao, S. Chem. Eur. J. 2011, 17, 12476.

[23] Cole, K. S.; Cole, R. H. J. Chem. Phys. 1941, 9, 341.

[24] Gatteschi, D.; Sessoli, R.; Villain, J. Molecular Nanomagnets, Oxford University Press, New York, 2006, p. 69. 